\title{
Aspectos epidemiológicos da sífilis gestacional em uma maternidade filantrópica e os desafios para seu controle
}

Analisar o perfil epidemiológico das puérperas acometidas pela sífilis em uma Maternidade Filantrópica de Aracaju (Sergipe) no período de outubro de 2018 a outubro de 2019. Trata-se de um estudo de caráter retrospectivo, transversal e descritivo, a partir de dados obtidos por meio de entrevistas realizadas pelos autores. O projeto foi aprovado pelo Comitê de Ética e Pesquisa da Universidade Tiradentes sob o parecer de número 2.922.433. Um total de 93 puérperas com VDRL (Venereal Disease Research Laboratory) positivo participaram do seguinte estudo. A idade média delas foi de 23,8 anos, 47 apresentavam ensino fundamental incompleto, 66 se autodeclaram cor parda, 62 eram donas de casa, 87 assintomáticas, 87 realizaram o pré-natal, 55 iniciaram no primeiro trimestre e 52 fizeram mais de 6 consultas. Houveram 65 puérperas que foram tratadas com Penicilina Benzatina, porém de forma inadequada. Foram encontradas 27 puérperas que já haviam tido pelo menos 1 aborto, 12 com feto morto, 92 só tiveram um parceiro sexual, 56 conviviam com eles e 42 foram tratados ao mesmo tempo das puérperas São necessárias medidas de saúde pública mais eficazes em relação a assistência ao pré-natal e educação da população capazes de reduzir a alta incidência da Sífilis.

Palavras-chave: Cuidado Pré-Natal; Epidemiologia; Gestantes; Sífilis.

\section{Epidemiological aspects of gestational syphilis in a philanthropic maternity and the challenges for control}

\begin{abstract}
Analysis of the epidemiologic profile of the mothers affected by syphilis in a Philanthropic Maternity in the city of Aracaju (Sergipe) whitin the period between October of 2018 and October of 2019. This is a prospective, cross-sectional and descriptive study from data selected through interviews conducted by the authors. The project was approved by the Ethics Committee on Human Research of Tiradentes University (№ 2.922.433). A total of 93 mothers who tested positive for VDRL (Venereal Disease Research Laboratory) participated. The average age was 23,8 years old, in which 47 did not conclude elementary school, 66 had brown skin, 62 were housewifes, 87 were asymptomatics, 87 took the prenatal care, 55 started in the first trimester of their pregnancy and 52 had more than 6 prenatal visits. There were 65 puerperal women who were treated with Benzathine Penicillin, however inappropriately. In 27 had already had at least 1 abortion, 12 had dead fetus, 92 only had one sexual partner during of their pregnancy, 56 having a relationship with them and 42 were treated at the same time the puerperal woman. More effective public health actions are necessary in prenatal care and in the education of the population in order to reduce the high incidence of Syphilis.
\end{abstract}

Keywords: Prenatal Care; Epidemiology; Pregnant Women; Syphilis.

Topic: Obstetrícia

Reviewed anonymously in the process of blind peer.
Received: 08/06/2020

Approved: 18/08/2020
Juliana Maria Dos Santos Cardoso (iD

Universidade Tiradentes, Brasil

http://lattes.cnpq.br/1976270854002203

http://orcid.org/0000-0002-0664-3045

julianamcardosoo@gmail.com

Marina Maria Santos Alves (id

Universidade Tiradentes, Brasil

http://lattes.cnpq.br/7013606234725882

http://orcid.org/0000-0001-9884-9608

marinaalves maria@hotmail.com

Izailza Matos Dantas Lopes (iD

Universidade Tiradentes, Brasil

http://lattes.cnpq.br/5911269328493167

http://orcid.org/0000-0001-9752-5628

izailzamatos@gmail.com
Israel Azevedo Siqueira de Carvalho

Universidade Tiradentes, Brasil

http://lattes.cnpq.br/9212803513641120

http://orcid.org/0000-0002-8208-1515

israelazevedose@gmail.com

Alex Vianey Callado França (iD

Universidade Tiradentes, Brasil

http://lattes.cnpq.br/6185407445596761

http://orcid.org/0000-0003-1362-2135

avcfranca@hotmail.com
Referencing this:

CARDOSO, J. M. S.; ALVES, M. M. S.; LOPES, I. M. D.; CARVALHO, I. A. S.; FRANÇA, A. V. C.. Aspectos epidemiológicos da sífilis gestacional em uma maternidade filantrópica e os desafios para seu controle. Scire Salutis, v.10, n.3, p.58-66, 2020. DOI:

http://doi.org/10.6008/CBPC2236-9600.2020.003.0007 


\section{INTRODUÇÃO}

A sífilis é uma doença crônica, sistêmica e tratável. Causada pela espiroqueta Treponema pallidum, sua transmissão é principalmente sexual, porém na gestante não tratada ou inadequadamente tratada cursa com o risco adicional de transmissão para o feto em qualquer fase da gestação e da doença (BRASIL, 2019; KUZNIK et al., 2015)

A Organização Mundial da Saúde (OMS) estima que a sífilis acometa um milhão de gestantes e no Brasil cerca de $\mathbf{3 0 0}$ mil casos por ano, acarretando mais de $\mathbf{3 0 0}$ mil mortes fetais e neonatais e risco de morte prematura em mais de 200 mil crianças (BRASIL, 2019; DOMINGUES et al., 2013). Em comparação ao ano de 2016, no Brasil observou-se aumento de $28,5 \%$ na taxa de detecção em gestantes (BRASIL, 2018). No Brasil, em 2017, foram notificados no SINAN (Sistema de Informação de Agravos de Notificação) 49.013 casos de sífilis em gestantes (taxa de detecção de 17,2/1.000 nascidos vivos) e em 2018, 62.599 casos de sífilis em gestantes (taxa de deteç̧ão de 21,4/1.000 nascidos vivos) (BRASIL, 2019).

Aproximadamente uma em cada três mulheres não são corretamente testadas para sífilis durante a gestação nos países asiáticos e latino-americanos (KUZNIK et al., 2015). De acordo com a Secretaria de Vigilância em Saúde, Sergipe é um dos seis Estados onde as taxas de incidência de sífilis congênitas são maiores que as de sífilis em gestante (BRASIL, 2019). Isso significa que possa estar havendo falhas na base: o pré-natal.

Esses dados alarmantes podem ser justificados pela maior propagação dos testes rápidos e assim da deteç̧ão dessa patologia, como também do decréscimo no uso de preservativos, e do desabastecimento global da penicilina (BRASIL, 2019). Por mais que existam condutas eficazes e de baixo custo para prevenir e controlar a sífilis, questões comportamentais e socioculturais impedem a adoção delas (OLIVEIRA et al., 2015). Além disso, Cerca de $14 \%$ das gestantes podem apresentar falha terapêutica, aumentando o risco de sífilis congênita (BRASIL, 2019).

Na gestação, a taxa de transmissão vertical pode ser de $70 \%$ a $100 \%$ se a gestante estiver na fase primária ou secundária da doença. A sífilis pode levar a graves sequelas para o feto como aborto, prematuridade, manifestações congênitas precoces ou tardias e/ou morte do recém-nascido (RN), entre 30 a 50\% (BRASIL, 2019).

Diante do cenário exposto, o seguinte estudo busca compreender o perfil epidemiológico das puérperas acometidas pela sífilis em uma Maternidade Filantrópica de Aracaju no período de outubro de 2018 a outubro de 2019. Teve como estímulo a alta incidência e prevalência da sífilis congênita no Estado de Sergipe tendo em vista investigar as raízes desse problema global: as mães para que os índices de transmissão vertical possam diminuir no futuro.

\section{METODOLOGIA}

Estudo epidemiológico de caráter retrospectivo, transversal e descritivo. Foi realizada uma coleta de dados no período de 1 ano, desde o período de outubro de 2018 a outubro de 2019, o que totalizaram 93 
puérperas com VDRL positivo. Essas eram abordadas e entrevistadas na UI (Unidade Intermediária) de uma maternidade filantrópica na cidade de Aracaju, do estado de Sergipe.

As variáveis analisadas foram idade materna, ocupação, escolaridade, quantidade de filhos, tempo de convivência com o parceiro e se possuíam outros filhos com ele, recebimento de bolsa família, quantidade de parceiros na gestação, sífilis em outras gestações, número de consultas e início do pré-natal, antecedentes obstétricos como a quantidade de gestações, partos e abortos, realização do VDRL e resultados desse, sintomatologia, tratamento da sífilis na gestação e pós-parto.

Foram incluídas todas as puérperas com VDRL positivo que seus conceptos estivessem vivos e internados na unidade intermediária da maternidade de estudo e que assinassem o Termo de Consentimento livre e esclarecido. Foram excluídas e não entrevistadas mães cujos conceptos apresentassem outras doenças cujos sintomas sejam semelhantes ao de sífilis congênita, como: Toxoplasmose, CMV, HIV, Rubéola, HVC e HBV.

Os dados foram analisados e interpretados utilizando a estatística descritiva e inferencial. As variáveis categóricas foram apresentadas por meio de frequências simples e relativas e as variáveis numéricas por meio de média e desvio padrão. O software utilizado para as análises foi o Statistical Package for the Social Sciences (IBM SPSS 25.0).

O projeto foi submetido ao Comitê de Ética em Pesquisa (CEP) da Universidade Tiradentes (UNIT), Sergipe, Aracaju, aprovado sob o parecer de número 2.922 .433 em 27 de setembro de 2018. Após a aprovação pelo CEP, iniciou-se a captação de indivíduos e coleta de dados. Todos os dados obtidos no presente estudo foram tabulados e resguardados, de forma a preservar qualquer identificação dos voluntários, conforme a resolução no 466/12, do Conselho Nacional de Saúde do Ministério da Saúde (CNS/MS). Os indivíduos que concordaram a participar do estudo, foram instruídos e receberam duas vias do Termo de Consentimento Livre e Esclarecido (TCLE), uma entregue aos participantes e a outra em posse dos pesquisadores, redigido de acordo com a resolução no 466/12 (CNS/MS).

\section{RESULTADOS}

A amostra contou com a participação de 93 puérperas, com VDRL positivo, que aceitaram ser entrevistadas na maternidade filantrópica de Aracaju. O perfil socioeconômico está demonstrado na Tabela 1. A média de idade das participantes foi de 23,8 (16-39) anos. O nível de escolaridade demonstrou que 50,5\% (47) possuíam ensino fundamental incompleto; $16,1 \%$ (15) com ensino fundamental completo; $16,1 \%$ (15) com ensino médio incompleto; $14 \%$ (13) com ensino médio completo e apenas 3,2\% (3) ensino superior escolaridade incompleto. Nenhuma delas apresentava ensino superior completo. Sobre a etnia, 71\% (66) declararam-se da cor parda; seguidas de 19,4\% (18) da cor negra; 6,5\% (6) da cor branca e apenas 1,1\% (1) da cor amarela. Cerca de 2,2\% (2) não informaram.

Ao voltar-se para as ocupações das puérperas, a que obteve destaque em preponderância foi a de dona de casa com 67\% (62). Das 25,8\% (24) demais eram Estudante (6,45\%), Vendedora (5,37\%), Comerciante (1,07\%), Lavradora/Agricultora (2,1\%), Manicure (2,1\%), Camareira (1,07\%), Garçonete $(1,07 \%)$, 
Cabelereira (1,07\%), Feirante (1,07\%), Depiladora (1,07\%), Autônoma (1,07\%), Animadora de Festas (1,07\%)). A quantidade de desempregadas foram 7,5\% (7). No tocante ao recebimento de bolsa família, 54,8\% (51) possuíam bolsa família Dados representados na Tabela 1.

Tabela 1: Perfil socioeconômico das 93 puérperas com VDRL positivo em uma maternidade filantrópica de Aracaju, outubro de 2018 a outubro de 2019.

\begin{tabular}{|c|c|}
\hline Variáveis & Frequência absoluta (relativa) \\
\hline \multicolumn{2}{|l|}{ Escolaridade } \\
\hline Fundamental incompleto & $47(50,5)$ \\
\hline Fundamental completo & $15(16,1)$ \\
\hline Médio incompleto & $15(16,1)$ \\
\hline Médio completo & $13(14)$ \\
\hline Superior incompleto & $3(3,2)$ \\
\hline Idade materna & $23,8 \pm 5,51$ \\
\hline \multicolumn{2}{|l|}{ Ocupação } \\
\hline Dona de casa & $62(67)$ \\
\hline Desempregada & $7(7,5)$ \\
\hline Outras ocupações & $24(25,8)$ \\
\hline \multicolumn{2}{|l|}{ Etnia } \\
\hline Branca & $6(6,5)$ \\
\hline Parda & $66(71)$ \\
\hline Negra & $18(19,4)$ \\
\hline Amarela & $1(1,1)$ \\
\hline Não informou & $2(2,2)$ \\
\hline \multicolumn{2}{|l|}{ Bolsa Família } \\
\hline Sim & $51(54,8)$ \\
\hline Não & $42(45,2)$ \\
\hline
\end{tabular}

Os dados numéricos estão exibidos em média \pm desvio-padrão. Os dados categóricos estão exibidos em frequência absoluta (frequência relativa). Fonte: Autoria própria.

Foi analisado também as características gestacionais das puérperas em questão. Os resultados encontrados estão registrados na Tabela 2. Sobre a quantidade de filhos, $29(31,2 \%)$ eram primigestas, 57 $(61,3 \%)$ tinham de 2 a 4 filhos e 7 (7,5\%) tinham 5 a 7 filhos. Acerca dos abortos, observou-se que 27 (29\%) puérperas já haviam tido pelo menos 1 aborto. Dessas, 23 (85,2\%) sofreram 1 aborto e 4 (14,8\%) 2 abortos. Em relação aos fetos mortos, 12 (12,9\%) puérperas já tiveram esse fenômeno, tendo 9 puérperas com 1 feto morto e 3 com 2 fetos mortos.

Também foi investigada a quantidade de parceiros na gestação e 98,9\% (92) afirmaram só terem se relacionado com um parceiro na gestação, o pai do recém-nascido em questão. A maioria, $56(60,21 \%)$ puérperas, relatou que ainda convivem com o pai do recém-nascido, com uma média de 4,5 anos, sendo o menor tempo 8 meses e o maior 16 anos. Sobre ter outros filhos com o pai do recém-nascido, 40 (43\%) puérperas afirmaram não ter tido, $32(34,4 \%)$ tiveram outros filhos e $21(22,6 \%)$ não informaram.

Tabela 2: Características gestacionais das 93 puérperas com VDRL positivo em uma maternidade filantrópica de Aracaju, outubro de 2018 a outubro de 2019

\begin{tabular}{|l|l|}
\hline Característica & Frequência absoluta (relativa) \\
\hline Número de filhos & \\
\hline Primigestas & $29(31,2)$ \\
\hline 2 a 4 filhos & $57(61,3)$ \\
\hline 5 a 7 filhos & $7(7,5)$ \\
\hline Número de abortos & \\
\hline 1 & $23(24,7)$ \\
\hline 2 & $4(4,3)$ \\
\hline
\end{tabular}




\begin{tabular}{|l|l|}
\hline Feto morto & \\
\hline Sim & $12(12,9)$ \\
\hline Não & $81(87,1)$ \\
\hline Quantidade de parceiros na gestação & \\
\hline 1 parceiro & $92(98,9)$ \\
\hline Mais de 1 parceiro & $1(1,1)$ \\
\hline Outros filhos com o pai do recém nascido & \\
\hline Nenhum & $40(43,0)$ \\
\hline 1 & $24(25,8)$ \\
\hline 2 & $3(3,2)$ \\
\hline 3 & $3(3,2)$ \\
\hline 4 & $2(2,2)$ \\
\hline Não informaram & $21(22,6)$ \\
\hline Média \pm desvio-padrão & $0,65 \pm 0,95$ \\
\hline
\end{tabular}

Os dados numéricos estão exibidos em média \pm desvio-padrão. Fonte: Autoria própria

Em relação ao pré-natal, 87 (93,5\%) puérperas realizaram, 5 (5,4\%) não realizaram e apenas 1 (1,1\%) não informou e não apresentava registro em prontuário. Relataram ter iniciado o pré-natal no primeiro trimestre 55 (63,21\%), 19 (21,83\%) no segundo trimestre e $3(3,44 \%)$ no terceiro trimestre. Porém não havia registro no cartão da gestante ou em prontuário 10 (11,5\%) puérperas. A média de consultas foi de 6,71, sendo 1 a menor quantidade e 13 a maior quantidade. Das que realizaram o pré-natal, 24 (27,6\%) puérperas tinham menos de 6 consultas, $52(59,8 \%)$ acima de 6 consultas e $11(12,6 \%)$ não tinham registros no cartão da gestante. Pode-se observar na Tabela 3.

Tabela 3: Características do pré-natal das 93 puérperas com VDRL positivo em uma maternidade filantrópica de Aracaju, outubro de 2018 a outubro de 2019

\begin{tabular}{|l|l|}
\hline Característica & Frequência absoluta (relativa) \\
\hline Realização do pré-natal & \\
\hline Sim & $87(93,5)$ \\
\hline Não & $5(5,4)$ \\
\hline Sem registro/ Não informado & $1(1,1)$ \\
\hline Período de início & \\
\hline 1 o trimestre & $55(63,21)$ \\
\hline 2o trimestre & $19(21,83)$ \\
\hline 3o trimestre & $3(3,44)$ \\
\hline Sem registro & $10(11,5)$ \\
\hline Total & $87(100)$ \\
\hline Número de Consultas & \\
\hline$<6$ consultas & $24(27,6)$ \\
\hline$\geq 6$ consultas & $52(59,8)$ \\
\hline Sem registro & $11(12,6)$ \\
\hline Total & $87(100)$ \\
\hline Média \pm desvio-padrão & $6,71 \pm 2,77$ \\
\hline
\end{tabular}

Os dados numéricos estão exibidos em média \pm desvio-padrão. Os dados categóricos estão exibidos em frequência absoluta (frequência relativa). Fonte: Autoria própria.

Algumas características acerca da sífilis nas puérperas entrevistadas podem ser observadas na Tabela 4. Observou-se que 42 puérperas $(45,2 \%)$, afirmaram não ter contraído a sífilis anteriormente em outras gestações, 20 (21,5\%) afirmaram já terem sido infectadas outras vezes e $31(33,4 \%)$ não sabiam informar. Sobre a sintomatologia da sífilis na amostra analisada, constatou-se que 92,5\% (87) eram assintomáticas. Das 6 puérperas que relataram ter tido algum sintoma, 50\% (3) afirmaram ter apresentado cancro duro e 50\% (3) manchas palmo-plantares eritematosas e acinzentadas descamativas. Os valores de VDRL no parto das puérperas demonstraram que $30(32,3 \%)$ tinham valores entre 1:1 a 1:4, $27(29 \%)$ entre 1:8 a 1:64 e 36 
(38,7\%) não tinham registro em prontuário.

Tabela 4: Características da Sífilis das 93 puérperas com VDRL positivo em uma maternidade filantrópica de Aracaju, outubro de 2018 a outubro de 2019

\begin{tabular}{|l|l|}
\hline Características & Frequência absoluta (relativa) \\
\hline Sífilis em outras gestações & \\
\hline Sim & $20(21,5)$ \\
\hline Não & $42(45,2)$ \\
\hline Não informado & $31(33,4)$ \\
\hline Sintomas da sífilis & \\
\hline Sem sintomas & $87(93,5)$ \\
\hline Cancro duro & $3(3,25)$ \\
\hline Manchas palmo-plantares eritematosas e acinzentadas & $3(3,25)$ \\
\hline Valores de VDRL & \\
\hline $1: 1$ a 1:4 & $30(32,3)$ \\
\hline $1: 8$ a 1:64 & $27(29)$ \\
\hline Sem registro & $36(38,7)$ \\
\hline
\end{tabular}

Os dados numéricos estão exibidos em média \pm desvio-padrão. Os dados categóricos estão exibidos em frequência absoluta (frequência relativa). Fonte: Autoria própria.

No quesito tratamento da sífilis, $65(69,9 \%)$ relataram terem realizado na gestação. Das $28(30,1 \%)$ que não realizaram o tratamento na gestação, 11 tinham VDRL não reagente, 8 informaram desconhecer que eram portadoras da sífilis, 4 não realizaram o VDRL durante a gestação, 3 não informaram o motivo e 1 relatou não ter tido interesse em realizar o tratamento. Após o parto, $53(56,98 \%)$ realizaram o tratamento e 40 (43\%) não realizaram. Constatou-se que 5 puérperas que não tinham realizado o tratamento durante a gestação também não realizaram o tratamento após o parto. Todas as puérperas que foram tratadas utilizaram a Penicilina Benzatina. Dados representados na Tabela 5.

Acerca do tratamento para sífilis nos pais dos neonatos, durante a gestação das puérperas, 49 $(52,7 \%)$ não o realizaram, $42(45,2 \%)$ realizaram com Penicilina Benzatina como também ao mesmo tempo que as participantes e $2(2,1 \%)$ não sabiam informar. Após o parto das puérperas, 7 (7,5\%) realizaram o tratamento com Penicilina Benzatina e ao mesmo tempo que as mães e $84(90,3 \%)$ não foram tratados.

Tabela 5: Tratamento da sífilis das 93 puérperas com VDRL positivo e dos seus parceiros em uma maternidade filantrópica de Aracaju, outubro de 2018 a outubro de 2019

\begin{tabular}{|l|l|}
\hline Realização de tratamento & Frequência absoluta (relativa) \\
\hline Tratamento da mãe & \\
\hline Sim & $65(69,9)$ \\
\hline Não & $28(30,1)$ \\
\hline Tratamento dos parceiros & \\
\hline Sim & $42(45,2)$ \\
\hline Não & $49(52,7)$ \\
\hline Não sabiam informar & $2(2,1)$ \\
\hline
\end{tabular}

Os dados categóricos estão exibidos em frequência absoluta (frequência relativa). Fonte: Autoria própria.

\section{DISCUSSÃO}

A sífilis continua sendo um problema mundial de saúde pública. Estudos nacionais revelaram que a assistência ao pré-natal no Brasil é universal e alcança valores superior a 90\%. Porém, a adequação dessa assistência é ainda baixa: 75,8\% das mulheres iniciaram o pré-natal até a 160 semana gestacional e apenas 73,1\% tiveram o número mínimo de consultas previstas para a idade gestacional no momento do parto (DOMINGUES et al., 2014; VIELLAS et al., 2020). 
No seguinte estudo, observou-se que 93,5\% das puérperas entrevistadas realizaram o pré-natal. Essa porcentagem ainda que alta, não está no número alvo. Uma das ações propostas pela OMS para o controle da sífilis na gestação é a realização de pelo menos uma consulta pré-natal com meta de realização de pelo menos $\geq 95 \%$ (WHO, 2017).

Destas, $63,2 \%$ relataram ter iniciado no primeiro trimestre, porém em $11,5 \%$ puérperas não havia registro do período do início dificultando averiguar dados do tempo correto da realização. Além disso, 27,6\% tiveram menos que 6 consultas e essa variável foi difícil estimar pois em 12,6\% não tinham registro em prontuário. No Brasil, recomenda-se no mínimo seis consultas, início idealmente no primeiro trimestre e condutas básicas a cada consulta (BRASIL, 2012). Um aspecto a ser analisado é que maior quantidade de consultas não significa necessariamente uma boa qualidade da assistência ao pré-natal (NUNES et al., 2016).

As características demográficas do presente estudo revelaram que a maioria das puérperas entrevistadas eram jovens, pardas, donas de casa, com ensino fundamental incompleto e nenhuma com ensino superior e recebiam bolsa família. Isso pode demonstrar que talvez exista uma desigualdade social em relação ao acesso e assistência à saúde. Aspectos sociais, econômicos, biológicos e culturais podem ser relevantes para a transmissibilidade da sífilis e persistência da transmissão vertical (PINTO et al., 2014)

De acordo com Lafetá et al. (2016), “a sífilis, assim como outras DST, não afeta apenas um grupo de risco específico, devendo ser fundamental a prevenção para a população em geral". Um estudo realizado no Pará relaciona o baixo nível socioeconômico ao pouco nível educacional que pode ser diretamente proporcional ao pouco conhecimento das Doenças Sexualmente Transmissíveis e a relevância dos cuidados pré-natais (ARAUJO et al., 2006). Ao olhar os motivos da não realização do tratamento, motivos como não conhecimento da patologia e o não interesse estavam envolvidos.

Observou-se que 92,5\% eram assintomáticas. O Ministério da Saúde refere que a maioria das pacientes com sífilis ocorrem na fase de latência, onde não se observam nenhum sinal ou sintomas e ao apresentar sinais ou sintomas não dão importância estando mais suscetíveis de transferir ao concepto e aos parceiros (BRASIL, 2019). Por isso, é importante a realização de testes diagnósticos, a exemplo do VDRL.

A titulação sorológica da infecção pode fornecer indicativos das condições fetais. Estudo conduzido por Nascimento et al. (2012) relataram que títulos do VDRL maior que 1:4 indicam infecção ativa, recentemente adquirida e com maior risco de envolvimento fetal, estando a mortalidade relacionada à intensidade da infecção, à resposta inflamatória e à redução do fluxo sanguíneo placentário. Na presente pesquisa, cerca de $29 \%$ tiveram títulos maiores que 1:4 durante o parto.

As puérperas tinham um tempo médio de convivência com o parceiro de 4,5 anos. Percebe-se que a confiança pode levar a uma negligência do uso dos métodos contraceptivos e assim uma maior chance de contrair a sífilis. Nesse estudo, observou-se que não houve o tratamento de $52,7 \%$ dos parceiros. O Ministério da Saúde, recomenda que todos os parceiros sexuais sejam tratados independente da titulação dos exames laboratoriais ou do quadro clínico (BRASIL, 2019). Além disso, o tratamento dos parceiros sexuais é imprescindível para uma diminuição das taxas de reinfecção na gestante (DOMINGUES et al., 2016).

A chance de infecção pela sífilis congênita é aumentada em cinco vezes em parceiros com sífilis. É 
fundamental que haja a participação dos homens no pré-natal pois um dos motivos da baixa adesão ao tratamento é a falta de conhecimento sobre a importância do tratamento para a saúde materna e infantil (SOARES et al., 2017).

Para o Ministério da Saúde, o tratamento adequado é considerado quando é por meio da penicilina benzatina de acordo com o estágio da sífilis, iniciado até 30 dias antes do parto, com dose da medicação de acordo com o estágio da doença e tratamento concomitante do parceiro (BRASIL, 2019). Percebeu-se que $(69,9 \%)$ foram inadequadamente tratadas visto que apenas $45,2 \%$ dos pais foram tratados e ao mesmo tempo da parceira. O diagnóstico e tratamento adequados diminuem a transmissão vertical em até $97 \%$ (DOMINGUES et al., 2014).

O único tratamento seguro e eficaz recomendado pelo Ministério da Saúde é com a benzilpenicilina benzatina (BRASIL, 2019). Essa foi a medicação de eleição para as puérperas que foram tratadas. Porém 30,1\% não havia sido tratada. O risco de contaminação pela sífilis congênita do feto e recém-nascido reduz para menos de $1 \%$ quando a mãe é adequadamente tratada (LOPES et al., 2018).

As consequências da infecção pela sífilis podem ser desastrosas. Em $40 \%$ dos casos de sífilis sem tratamento levam ao aborto espontâneo e sem tratamento adequado ocorrem $11 \%$ de mortes fetais a termo (BRASIL, 2019). No presente estudo, observou-se que 29\% já abortaram e 12,9\% tiveram pelo menos 1 feto morto. Um entrave na realização do seguinte estudo foi o não preenchimento de alguns dados no cartão da gestante de algumas puérperas. Torna-se necessário a capacitação dos profissionais de saúde para o entendimento do real problema e propor intervenções mais eficazes (COSTA et al., 2013).

\section{CONCLUSÕES}

O perfil epidemiológico de puérperas com sífilis gestacional na maternidade filantrópica de Aracaju é predominantemente em jovens, de cor parda, donas de casa, com ensino fundamental incompleto e que recebem bolsa família. A maioria era assintomática, tinha realizado o pré-natal, e sido tratadas com Penicilina Benzatina, como preconiza o Ministério da Saúde, porém de forma inadequada. Alguns prejuízos trazidos para os conceptos, no seguinte estudo, foram aborto e feto morto. A maior parte convivia com o pai do recém-nascido em questão e percebeu-se que o não tratamento por parte deles pode ser determinante para a transmissão vertical da mãe para o concepto.

Além disso, é importante salientar que é necessário a tomada de medidas de controle como a maior instrução para a população e capacitação aos profissionais de saúde. Por fim, estudos com uma maior amostra no Estado de Sergipe podem ser realizados para um melhor esclarecimento dos desafios do manejo dos casos.

\section{REFERÊNCIAS}

ARAUJO, E. D. C.; COSTA, K. D. S. G.; SILVA, R. D. S. E.; AZEVEDO, V. N. D. G.; LIMA, F. A. S.. Importância do prénatal na prevenção da Sífilis Congênita. Rev. Para. Med., Belém, v.20, n.1, p.47-51, 2006.
BRASIL. Ministério da Saúde. Atenção ao pré-natal de baixo risco. Brasília: Ministério da Saúde, 2012.

BRASIL. Ministério da Saúde. Boletim Epidemiológico: Sífilis. Brasília: Secretaria de Vigilância em Saúde, 2018. 
BRASIL. Ministério da Saúde. Protocolo Clínico e Diretrizes Terapêuticas para Atenção Integral às Pessoas com Infecções Sexualmente Transmissíveis (IST). Brasília: Ministério da Saúde, 2019.

COSTA, C. C. D.; FREITAS, L. V.; SOUSA, D. M. D. N.; OLIVEIRA, L. L. D.; CHAGAS, A. C. M. A.; LOPES, M. V. D. O.; DAMASCENO, A. K. C.. Sífilis congênita no Ceará: análise epidemiológica de uma década. Rev. Esc. Enferm. USP, São Paulo, v.47, n.1, p.152-159, 2013. DOI: https://doi.org/10.1590/S0080-62342013000100019

DOMINGUES, R. M. S. M.; LEAL, M. D. C.. Incidência de sífilis congênita e fatores associados à transmissão vertical da sífilis: dados do estudo Nascer no Brasil. Cad. Saúde Pública, Rio de Janeiro, v.32, n.6, p.e00082415, 2016. DOI: https://doi.org/10.1590/0102-311X00082415

DOMINGUES, R. M. S. M.; SARACEN, V.; HARTZ, Z. M. D. A.; LEAL, M. D. C.. Sífilis congênita: evento sentinela da qualidade da assistência pré-natal. Rev. Saúde Pública, São Paulo, v.47, n.1, p.147-157, 2013. DOI: https://doi.org/10.1590/\$0034-89102013000100019

DOMINGUES, R. M. S. M.; SZWARCWALD, C. L.; SOUZA JUNIOR, P. R. B.; LEAL, M. D. C.. Prevalência de sífilis na gestação e testagem pré-natal: Estudo Nascer no Brasil. Rev. Saúde Pública, São Paulo, v.48, n.5, p.766-774, 2014. DOI: https://doi.org/10.1590/S0034-8910.2014048005114

KUZNIK, A.; ABDULRAZAQ, G. H.; YUKARI, C. M.; MOHAMMED, L.. Estimating the Public Health Burden Associated With Adverse Pregnancy Outcomes Resulting From Syphilis Infection Across 43 Countries in Sub-Saharan Africa. Sex Transm. Dis., Washington, v.42, n.7, p.369-375, 2015. DOI: https://doi.org/10.1097/OLQ.0000000000000291

LAFETÁ, K. R. G.; MARTELLI JÚNIOR, H.; SILVEIRA, M. F.; PARANAÍBA, L. M. R.. Sífilis materna e congênita, subnotificação e difícil controle. Rev. Bras. Epidemiol., São Paulo, v.19, n.1, p.63-74, 2016. DOI: https://doi.org/10.1590/1980-5497201600010006

LOPES, I. M. D.; LOPES, A. D.; SANTOS, R. S.; LIMA, S. O.; REIS, F. P.. Congenital syphilis in a philanthropic maternity of the state of Sergipe: still a challenge. DST Braz. J. Sex.
Transmitted Dis., Niteroi, v.30, n.2, p.41-46, 2018. DOI: https://doi.org/10.5533/DST-2177-8264-201830202

NASCIMENTO, M. I. D.; CUNHA, A. D. A.; GUIMARÃES, E. V.; ALVAREZ, F. S.; OLIVEIRA, S. R. D. S. M.; BÔAS, E. L. V.. Gestações complicadas por sífilis materna e óbito fetal. Rev. Bras. Ginecol. Obstet., Rio de Janeiro, v.34, n.2, p.56-62, 2012. DOI: https://doi.org/10.1590/S010072032012000200003

NUNES, J. T.; GOMES, K. R. O.; RODRIGUES, M. T. P.; MASCARENHAS, M. D. M.. Qualidade da assistência pré-natal no Brasil: revisão de artigos publicados de 2005 a 2015. Cad. Saúde Colet., Rio de Janeiro, v.24, n.2, p.252-261, 2016. DOI: https://doi.org/10.1590/1414-462X201600020171

OLIVEIRA, L. R. D.; COSTA, M. D. C. N.; BARRETO, F. R.; PEREIRA, S. M.; DOURADO, I.; TEIXEIRA, M. G.. Evaluation of preventative and control measures for congenital syphilis in State of Mato Grosso. Rev. Soc. Bras. Med. Trop., Uberaba, v.47, n.3, p.334-340, 2014. DOI: https://doi.org/10.1590/0037-8682-0030-2014

PINTO, V. M.; TANCREDI, M. V.; ALENCAR, H. D. R. D.; CAMOLESI, E.; HOLCMAN, M. M.; GRECCO, J. P. GRANGEIRO, A.; GRECCO, E. T. O.. Prevalência de Sífilis e fatores associados a população em situação de rua de São Paulo, Brasil, com utilização de Teste Rápido. Rev. Bras. Epidemiol., São Paulo, v.17, n.2, p.341-354, 2014. DOI: https://doi.org/10.1590/1809-4503201400020005ENG

SOARES, L. G.; ZARPELLON, B.; SOARES, L. G.; BARATIERI, T.; LENTSCK, M. H.; MAZZA, V. D. A.. Sífilis gestacional e congênita: características maternas, neonatais e desfecho dos casos. Rev. Bras. Saúde Mater. Infant., Recife, v.17, n.4, p.781-789, 2017. DOI: https://doi.org/10.1590/180693042017000400010

VIELLAS, E. F.; DOMINGUES, R. M. S. M.; DIAS, M. A. B.; GAMA, S. G. N. D.; THEME FILHA, M. M.; COSTA, J. V. D. BASTOS, M. H.; LEAL, M. C.. Assistência pré-natal no Brasil. Cad. Saúde Pública, Rio de Janeiro, v.30, n.1, p.S85-S100, 2014. DOI: http://dx.doi.org/10.1590/0102-311X00126013

WHO. World Health Organization. Global guidance on criteria and processes for validation: elimination of motherto-child transmission of HIV and syphilis. 2 ed. Geneva: World Health Organization, 2017.

A CBPC - Companhia Brasileira de Produção Científica (CNPJ: 11.221.422/0001-03) detém os direitos materiais desta publicação. Os direitos referem-se à publicação do trabalho em qualquer parte do mundo, incluindo os direitos às renovações, expansões e disseminações da contribuição, bem como outros direitos subsidiários. Todos os trabalhos publicados eletronicamente poderão posteriormente ser publicados em coletâneas impressas sob coordenação da Sustenere Publishing, da Companhia Brasileira de Produção Científica e seus parceiros autorizados. Os (as) autores (as) preservam os direitos autorais, mas não têm permissão para a publicação da contribuição em outro meio, impresso ou digital, em português ou em tradução. 